\title{
PET FrONTEIRAS - Saberes e Práticas Populares: uma proposta pautada na diversidade social e cultural
}

\author{
PET BORDERS - Popular Knowledge and Practices: a proposal guided by \\ the social and cultural diversity
}

\author{
PET FRONTERAS - Conocimientos y Prácticas Populares: una propuesta \\ guiada por la diversidad social y cultural
}

\begin{abstract}
Resumo
Esse trabalho pretende expor a proposta de pensar e agir do PET Fronteiras: Saberes e Práticas Populares, bem como os projetos e ações que estão inseridos nessa proposta que pauta uma educação voltada à diversidade e a cultura. Ao se pensar nas práticas e saberes populares, busca-se a produção de conhecimento a partir das manifestações culturais e populares das comunidades urbanas da cidade de Pelotas/RS, interagindo entre o conhecimento das comunidades populares e a universidade. Nosso objetivo se pauta em apresentar essas ações desenvolvidas pelo PET Fronteiras onde a experimentação artística dá-se como condição de um fazer educativo pelos caminhos da diversidade social e cultural.
\end{abstract}

Palavras-Chave: Pedagogia da Fronteira; educação; comunidades urbanas, diversidade e cultura.

\begin{abstract}
This work intends to present the proposal of thinking and acting of PET Borders: Knowledge and Practices Popular, as well as projects and actions that are included in this proposal that guides an education focused on diversity and culture. When you think of the popular practices and knowledge, seeks the production of knowledge from the cultural and popular manifestations of urban communities in the Pelotas city, interacting with knowledge of grassroots communities and the university. Our goal is guided to submit such actions developed by PET Borders where artistic experimentation is given as a condition of an education to the path of social and cultural diversity.
\end{abstract}

Keywords: Border pedagogy; education; urban communities, diversity and culture.

\section{Resumen}

Este trabajo tiene la intención de presentar la propuesta de pensar y actuar de la PET Fronteras: conocimientos y prácticas populares, así como los proyectos y acciones que se inserta en la propuesta que guía a una educación centrada en la diversidad y la cultura. Cuando pensamos en las prácticas y saberes populares, busca la 
producción de conocimiento de las manifestaciones culturales y populares de las comunidades urbanas en la ciudad de Pelotas / RS, interactuando con el conocimiento de las comunidades de base y la universidad. Nuestro objetivo es guiado a presentar este tipo de acciones desarrolladas por PET Fronteras donde se da la experimentación artística como condición de una educación para la trayectoria de la diversidad social y cultural.

Palabras claves: La pedagogía de frontera; la educación; comunidades urbanas, la diversidad y la cultura.

\section{Introdução}

O PET - Programa de Educação Tutorial - Fronteiras, é um PET de abrangência institucional da Universidade Federal de Pelotas - UFPel e compõe a proposta do PET Conexões e Saberes desenvolvida pelo Ministério da Educação, que busca como ação a troca de conhecimentos entre as comunidades populares urbanas e a academia. Atualmente o PET Fronteiras conta com 12 bolsistas de diversas áreas do conhecimento (ciências sociais, ciência da computação, teatro, geografia, direito, relações internacionais, direito, música e jornalismo).

Desse modo, o PET Fronteiras tem como objetivo tematizar os saberes e práticas populares focalizando a produção de conhecimentos verificados através das manifestações culturais que se desenvolvem nas comunidades populares urbanas da cidade de Pelotas, visando à interlocução com o que é produzido pela universidade. Sustentando-se por uma proposta de educação voltada à diversidade social e ao respeito aos direitos humanos como princípios nos marcos que os estudos culturais conferem como sendo o de uma Pedagogia da Fronteira.

O conceito de fronteira, segundo Bussoletti e Vargas (2014, p. 42) é "um elemento fundamental para pensarmos em propostas pedagógicas que abarquem as diferenças como um foco de aprofundamento ao enriquecimento da produção do conhecimento em sua ampla complexidade". Sendo que é nesse conceito de pedagogia da fronteira que fomentam-se as propostas pedagógicas do grupo, abarcando as diferenças como práticas educativas e emancipatórias dentro do cenário educativo brasileiro.

Além disso, necessitamos situar que o PET Fronteiras integra o Núcleo de Arte Linguagem e Subjetividade - NALS, vinculado a Faculdade de Educação (FaE) da UFPel, e que atualmente conta com 4 bolsistas (das áreas do teatro e psicologia) e diversos colaboradores. O NALS é um núcleo de extensão que busca, principalmente, aliar pesquisa e 
intervenção, utilizando-se da experimentação artística como modo de educar, trilhando os caminhos da diversidade, acreditando na extensão como forma de aproximação entre a universidade e a sociedade, utilizando-se fundamentalmente de armas culturais para promover o exercício da cidadania e a responsabilidade social.

O núcleo tem como base o conceito de extensão formulado pelo Fórum Nacional de Pró-Reitores 1987-2000, que entende a extensão universitária como um "processo educativo, cultural e científico que articula o ensino e a pesquisa de forma indissociável e viabiliza a relação transformadora entre a universidade e a sociedade" (NOGUEIRA, 2000, p. 11). O PET Fronteiras integra esse núcleo e busca também, em suas ações, a indissociabilidade entre o ensino, a pesquisa e a extensão. PET Fronteiras e NALS caminham juntos, desenvolvendo suas ações lado a lado.

Existente desde 2008, o NALS traz consigo dois pressupostos que regem sua atuação; o primeiro, a construção de um conhecimento científico que se afirme de outra maneira, e o segundo essencialmente metodológico, que caracteriza sua ação como a união de técnicas educativas e experimentos.

Propomos assim, uma reflexão sobre uma das ações desenvolvidas pelo PET Fronteiras, juntamente com o NALS, em 2014, na qual tem-se como base a experimentação artística, buscando essa proposta pedagógica da diversidade e a troca de saberes através de manifestações culturais e populares.

\section{O PET Fronteiras e suas ações}

O objetivo das ações do PET Fronteiras sustentam-se na perspectiva de uma educação voltada a diversidade social e cultural como já exposto acima, buscando atuar na construção de um processo de formação que preze pela indissociabilidade do ensino, pesquisa e a extensão de forma crítica e participativa, assegurando o elevado padrão de qualidade e de excelência, possibilitando desta forma também o sucesso acadêmico e a conclusão dos cursos aos quais se vinculam os estudantes oriundos das comunidades populares em condições de vulnerabilidade social e econômica (PROGRAMA DE EDUCAÇÃO TUTORIAL - PET FRONTEIRAS, 2012). 
O conjunto das ações se verificara basicamente através de três projetos: um projeto de ensino intitulado "aula extra", que pretende contribuir para promover o sucesso acadêmico e a conclusão dos cursos aos quais se vinculam os estudantes oriundos das comunidades populares, constituindo um espaço institucional de ensino direcionados às necessidades pedagógicas emergentes. Um projeto de pesquisa, "Narrativas e Saberes Populares", que busca tematizar os saberes e as práticas populares focalizando a produção de conhecimentos verificados através das manifestações culturais que se desenvolvem nas comunidades populares urbanas e rurais, promovendo a articulação com os conhecimentos produzidos na universidade, a fim de incrementar a produção acadêmica acerca dos saberes e práticas populares, buscando também inventariar os principais eventos e atividades populares e culturais criando então uma "Agenda/Catálogo Cultural" na possibilidade de incentivar veículos de informação entre as comunidades populares e a universidade na perspectiva de ações afirmativas de inclusão social. E um projeto de extensão denominado "parceria", que pretende evitar a evasão e contribuir para a redução das taxas de reprovação por frequência e de retenção, instituindo ações de acompanhamento de oferta de apoio pedagógico aos estudantes (PROGRAMA DE EDUCAÇÃO TUTORIAL - PET FRONTEIRAS, 2012).

É também importante localizar que os pressupostos pedagógicos do PET Fronteiras se baseiam através daquilo que os estudos culturais definem como uma "pedagogia da fronteira" (Giroux, 1992), ou ainda no que Mclarem (2000) denominou como sendo uma "identidade de fronteira" criadas a partir da empatia [...] como forma de uma conexão passional através da diferença "na luta" contra a nossa falha em ver nosso próprio reflexo nos olhos dos outros" (MCLAREM, 2000, p.194-195).

\section{As ações em práticas: tirando as "máscaras"}

Diversas foram as ações e atividades desenvolvidas pelo PET Fronteiras no decorrer de 2014, e muitas dessas ações perpassam pelo campo das artes, buscando nela uma forma de atuação e reflexão para o desenvolvimento de nossas principais atividades, que visam nossa percepção dentro da sociedade e a mudança de paradigmas (BUSSOLETTI; VARGAS, 2014, p. 41).

Nesse momento iremos apresentar a performance das "Máscaras" que situa-se na linha de "Narrativas e Saberes Populares" que busca tematizar os saberes e as práticas populares 
focalizando a produção de conhecimentos verificados através das manifestações culturais que se desenvolvem nas comunidades populares urbanas e rurais, promovendo a articulação com os conhecimentos produzidos na universidade a fim de incrementar a produção acadêmica acerca dos saberes e práticas populares. Assim, através das “máscaras” propomos contar uma outra história sobre a cidade de Pelotas, uma história muitas vezes esquecida, mas carregada de significados.

Essa performance foi desenvolvida em dois momentos: primeiramente fizemos a confecção das máscaras em novembro de 2014, para a realização do lançamento do III Forúm Internacional de Contadores de Histórias ${ }^{1}$. As máscaras foram moldadas com gesso em nossos rostos, com ajuda dos demais bolsistas, e depois as colorimos, dando-as emoção, cores e personalidade.

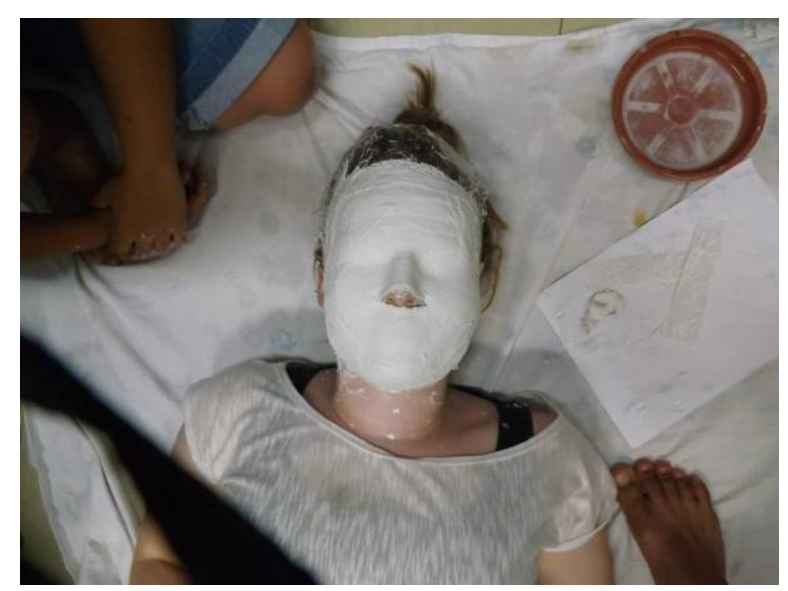

Figura 1. Confecção das máscaras. Fonte: Acervo NALS

\footnotetext{
${ }^{1}$ O primeiro Forúm Internacional de Contadores de Histórias ocorreu em 2009, em Pelotas, organizado pelo NALS. O evento contou com diversas representações dos movimentos sociais e dos grupos populares, possibilitando a troca de experiências através das contações de histórias, entre os narradores e o público. De acordo com Pinheiro e Bussoletti (2011, p. 237), os "narradores tinham total liberdade narrar suas histórias" e o que "interessava ao Forúm eram as histórias contadas, as quais permitiam a valorização desse instrumento de expressão da cultura popular e da permanência da memória".
} 


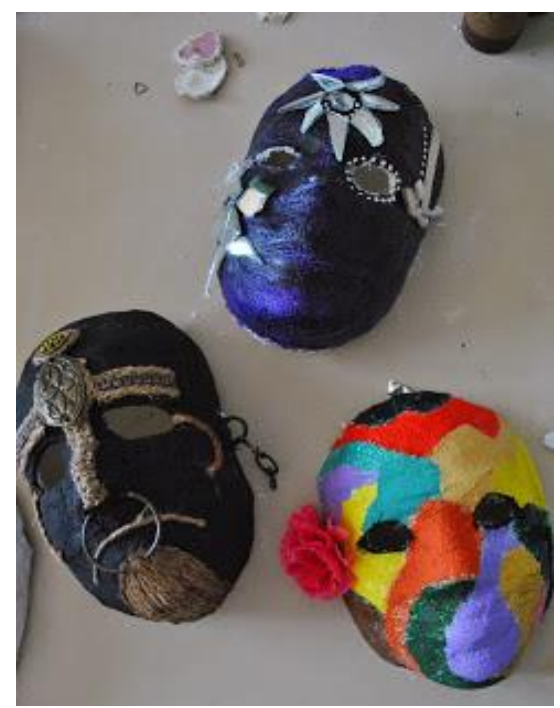

Figura 2. As máscaras. Fonte: Acervo NALS

Desse modo a confecção e performance das máscaras foi realizada com a finalidade de nos apresentarmos, de tirarmos nossas "máscaras" e contarmos nossas histórias. Contar sobre o lugar de onde viemos, contar uma outra história, aquela que muitas vezes não é contata, que passa abatida e que muitas vezes não temos tempo de parar e ouvir o que o "outro" tem a nos dizer.

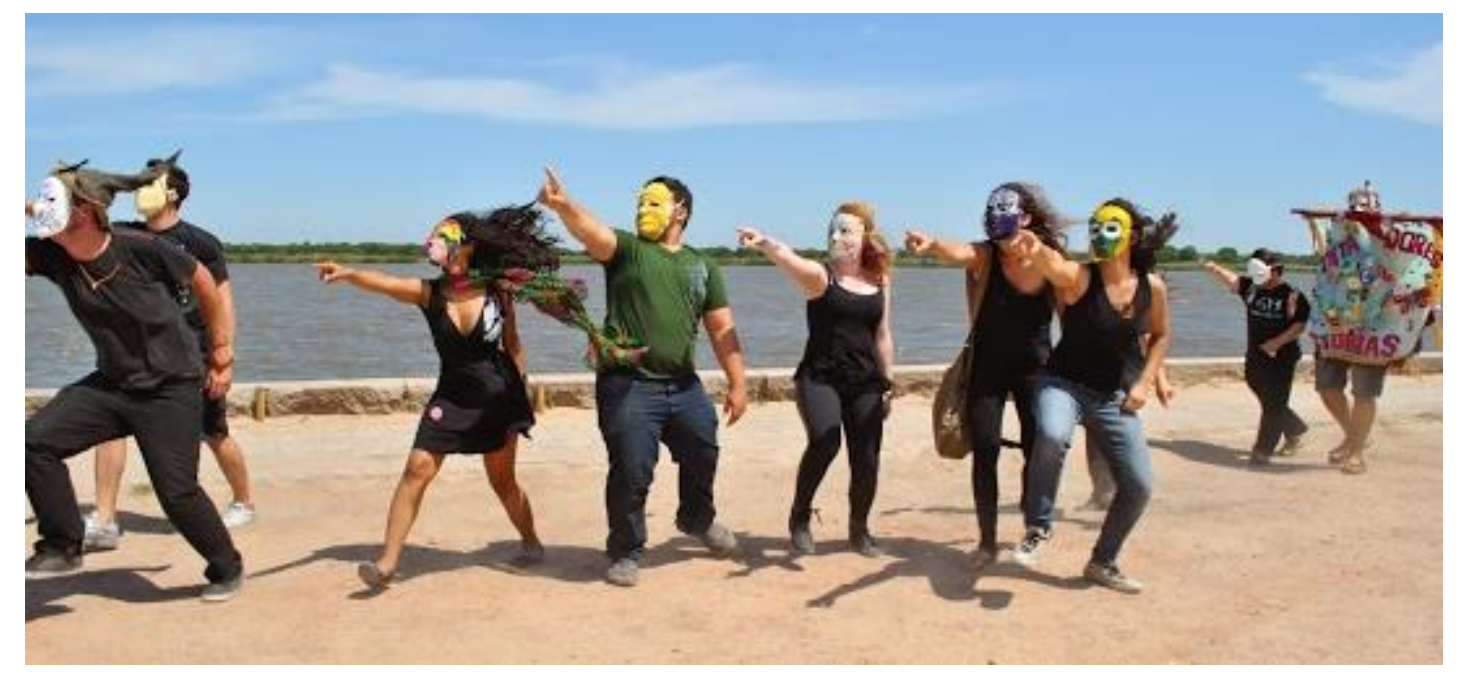

Figura 3. Performance das máscaras. Fonte: Acervo NALS 
No segundo momento, descolando-nos pelo Quadrado, região situada no bairro Porto de Pelotas, dando inicio a performance, como um jogo, uma brincadeira como o do "siga o mestre", assumindo uma dimensão subjetiva de nós mesmo pela busca dessas histórias, ou das nossas próprias histórias. Pautados nessa educação do sensível, da troca com o "outro" (CLIFFORD, 2008), permeamos por esse caminho que agora nos possibilita tirar as "máscaras".

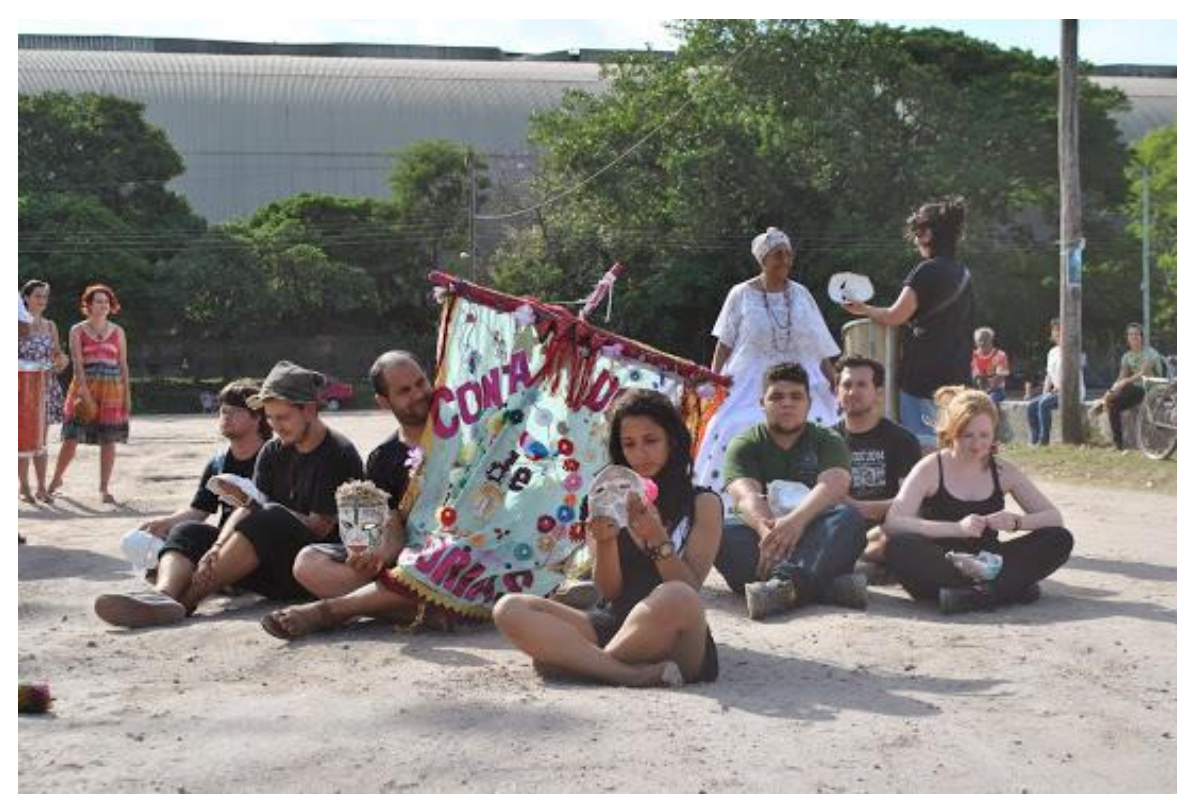

Figura 4. Tirando as "máscaras". Fonte: Acervo NALS

Ao "tirar" nossas "máscaras" e contarmos nossas histórias, propomos que as pessoas que se fizeram presentes no local da realização da performance, também compartilhassem suas histórias. Assim, temos a experiência dessa performance como um disparador para um diálogo propondo a troca de conhecimentos entre a comunidade acadêmica e as comunidades populares, promovendo o respeito, a diversidade, a cultura e o conhecimento dos saberes e práticas populares dentro do espaço educativo.

\section{Considerações finais}

Educar na diversidade, respeitando a identidade de cada um, aceitando as diferenças na perspectiva da igualdade entre os mesmos, é quase um "lugar comum" em se tratando das 
práticas discursivas atuais na educação. No entanto, o PET Fronteiras ao refletir os saberes e as práticas das comunidades populares busca refletir e agir enfrentando as lacunas importantes dos processos educativos nos marcos das fronteiras e das mobilizações que se verificam em torno da igualdade na diferença e da diferença na igualdade em sua multiplicidade de sentidos.

O PET Fronteiras: Saberes e Práticas Populares alicerça-se naquilo que Heidegger apud Bhabha (1998, p. 19) afirma quando diz que: "Uma fronteira é o ponto a partir do qual algo começa e se fazer presente."

\section{Referências}

BUSSOLETTI, Denise Marcos; VARGAS, Vagner de Souza. Por entre fronteiras de uma pedagogia que pauta a educação pelas artes gingando saberes e práticas populares.

Extraprensa, São Paulo, ano 8, nº 4, p. 41-48, 2014.

CLIFFORD, James. A experiência etnográfica: antropologia e literatura no século XX. Rio de Janeiro: Editora UFRJ, 2008.

GIROUX, H. Border Crossing. Nova York E Londres, Routledge, 1992.

HEIDEGGER, M. Poetry, Language, Trought. New York Harper e Row, 1971. Building, dwelling, thinking. In: BHABHA, H. O Local da Cultura. Belo Horizonte: Editora UFMG, 1998.

PINHEIRO, Cristiano Guedes; BUSSOLETTI, Denise Marcos. Terceira margem: um entrelugar nos novos espaços de fronteiras - as narrativas populares. In: BUSSOLETTI, D. M.; CANAL, C. Y.; GUEVARA, A. E.; LANDÍN, D. M. (orgs.). Pluralismo nas Ciências Sociais: da multiplicidade à diferença. Pelotas: Editora Universitária/UFPel, 2011.

PORTAL DO MINISTÉRIO DA EDUCAÇÃO (MEC). Disponível em: <http://portal.mec.gov.br>. Acessado em: 26 outubro 2015.

PROGRAMA DE EDUCAÇÃO TUTORIAL - PET FRONTEIRAS: Saberes e Práticas Populares, Universidade de Federal de Pelotas, 2012.

NOGUEIRA, Maria das Dores Pimentel (org). Extensão Universitária: diretrizes conceituais e políticas - Documentos básicos do Fórum Nacional de Pró-Reitores de Extensão das Universidades Públicas Brasileiras 1987 - 2000. Belo Horizonte: PROEX/UFMG, 2000.

MCLAREN, P. Multiculturalismo Crítico. São Paulo: Cortez, 1999. 